\title{
The relationship between blood pressure variability and outcome in acute ischemic stroke
}

\author{
Kamil Kowalczyk $\bullet$, Dariusz Gąsecki
}

Department of Adult Neurology, Medical University of Gdańsk, Poland

\begin{abstract}
Stroke is the second most common cause of mortality. Ischemic stroke is approximately 10 times more common than haemorrhagic stroke. The strongest risk factor for ischemic stroke is hypertension, thus reduction of blood pressure decreases the risk of ischemic stroke. However, the prognostic importance of blood pressure after is unclear. The problem is even more complex considering blood pressure variability (BPV), i.e. continuous changes of blood pressure values. The aim of this review is to discuss the very short-term, short-term, mid-term, and long-term blood pressure variability in the context of clinical outcome in patients after acute ischemic stroke. Most of the studies have shown that increased BPV in ischemic stroke patients is associated with poorer prognosis, however in some of them there was not association between BPV and outcome.
\end{abstract}

Keywords: outcome $\cdot$ ischemic stroke $\cdot$ blood pressure variability

\section{Citation}

Kowalczyk K, Gąsecki D. The relationship between blood pressure variability and outcome in acute ischemic stroke. Eur J TransI Clin Med. 2019;2(2):61-70.

DOI: $10.31373 /$ ejtcm/115986

\section{Introduction}

Worldwide, stroke is the second most common cause of death, after ischemic heart disease, and the second most common cause of reduced disability-adjusted life-years [1-3]. In Poland in 2009-2013, an annu- al hospital admission rate of ischemic stroke patients (standardized by age) is $127 / 100,000$ for females and $193 / 100,000$ for males [4]. Hypertension is the strongest risk factor for ischemic stroke. In the INTERSTRO-

Corresponding author:

Kamil Kowalczyk, Department of Adult Neurology, Medical University of Gdańsk, Poland

e-mail: kkowalczyk@gumed.edu.pl

No external funds.

Available online: www.ejtcm.gumed.edu.pl

Copyright ${ }^{\circledR}$ Medical University of Gdańsk

This is Open Access article distributed under the terms of the Creative Commons Attribution-ShareAlike 4.0 International. 
KE study, self-reported history of hypertension occurred in $31.5 \%$ of patients with acute ischemic stroke (AIS), while the incidence of hypertension defined as self-reported history of hypertension or blood pressure (BP) > 160/90 mm Hg was even higher (45.2\%) [5]. No Polish nation-wide data on the hypertension incidence in ischemic stroke patients are available, however in the years 2013-2014 the pre-hospital incidence of hypertension in Szczecin was $84.9 \%$ and $81.2 \%$ in Kraków \{Formatting Citation\}. Consequently, hypertension management reduces the incidence of stroke and therefore is recommended in primary prevention of ischemic stroke [8-10]. However, the prognostic significance of blood pressure in determining prognosis of stroke patients is unclear. The relationship between outcome and blood pressure often is U-shaped - both high and low early blood pressures may be associated with poorer outcome, yet the data results are inconsistent [11-13].

Furthermore, the management of altered BP in AIS remains challenging. Recommendations included in the current guidelines on BP treatment in AIS patients are not evidence-based [10, 15]. Present data suggest that lowering very high blood pressure in the early phase of AIS may be beneficial, but they need further confirmation. Data on raising blood pressure in the case of hypotension associated with focal cerebral ischemia are also inconsistent. Thus, due to insufficient data there is a continued concern about management of BP in patients with AIS [14]. Moreover, high blood pressure should be lowered for the purpose of secondary prevention after the acute phase of ischemic stroke or transient ischemic attack $[10,15]$.

Despite all the controversies, the role of BP and its management in acute stroke seems to be meaningful $[13,16-18]$. However, not only casual BP measurements affect the prognosis after AIS, but also BP profiles may be an important factor. Evidence concerning their role in ischemic stroke is scarce.

There is a paucity of the available data on the course of BP in ischemic stroke. In the vast majority of patients, during the acute phase of ischemic stroke blood pressure usually rises during the first hours and then declines over the time (a phenomenon known as acute hypertensive response, occurring in over $60 \%$ of such patients) [19-20]. Blood pressure upon admission is higher in patients with a history of hypertension [21]. Moreover, decrease in BP during the first 4 hours after admission is seen in patients with mild stroke and with subsequent favourable outcome [21]. On the contrary, no decline in BP is observed in patients with severe stroke and unfavourable outcome [21].
The variation in blood pressure over time is known as blood pressure variability (BPV) [22]. The role of BPV in ischemic stroke is poorly understood. BPV was found to be higher in ischemic stroke patients receiving antihypertensives (without specifying the drug class) just before administration of thrombolytics [23]. More precisely, calcium-channel blockers decrease BPV and reduce the risk of stroke [24]. Longitudinal observations of the general population in Japan showed that increased BPV was associated with higher stroke mortality [25]. The aim of this review is to discuss the role of BPV in prognosis after ischemic stroke. Other interesting factors that may influence ischemic stroke outcome are changes of blood pressure during the sleep/ wake cycle, however the topic is beyond the scope of this review and requires further study.

\section{Indices of blood pressure variability}

Blood pressure changes substantially between beats (very short-term BPV); minutes, hours or from day to night (within 24 hours, short-term BPV); between different days (mid-term BPV) or between clinic visits (long-term BPV) [26-27]. Indices used for assessment of BPV are as follows: standard deviation (SD), coefficient of variation (CV, defined as average SD divided by the mean BP and multiplied by 100), variation independent of mean (VIM, a transformation of SD which is not correlated with mean), average real variability (ARV, defined as the average of the absolute differences between successive BP measurements), successive variation (SV, defined as the square root of the average squared difference between consecutive BP measurements) [26, 28-30]. Novel approach includes more advanced methods, such as spectral analysis of the different frequency components [26, 31].

\section{Patomechanism}

\section{of blood pressure variability}

There are several mechanisms having an impact on BPV; e.g., very short- and short-term BPV is caused by humoral, rheological, behavioural, and emotional factors; impact of reflex and central autonomic modulation or arterial elasticity; mid- and long-term BPV, by behavioural factors, arterial stiffness, as well as seasonal climatic change, or poor control of blood pressure in treated patients [26]. Detailed description of the BPV pathomechanism is beyond the scope of this review and may be found elsewhere [32-33]. 


\section{Very short-term blood pressure variability}

Dawson et al assessed beat-to-beat BPV in 92 acute stroke patients [34]. The subjects had Finapres non-invasive BP monitor fitted within 24 to 72 hours after symptom onset. SD of beat-to-beat recordings (SBP, DBP, pulse pressure and mean arterial pressure) was calculated. The patients were classified as dead/ dependent or independent (mRS $\leq 2$ points) on the basis of assessment at 30 days after stroke. Patients with higher DBP and mean arterial pressure variability were of greater risk of poor outcome.

\section{Short-term blood pressure variability}

In a Spanish study, Delgado-Mederos et al assessed the impact of BPV on enlargement of diffusion-weighted imaging (DWI) ischemic lesion and clinical outcome [35]. 80 stroke patients with occlusion of middle cerebral artery, treated with intravenous tissue plasminogen activator (tPA) were included into the study. All of them had DWI before and 36-48 hours after thrombolysis. Repeated SBP and DBP measurement were recorded for 24 hours since admission. BP variability was defined as SD of all BP measurements. In non-recanalized patients, SBP and DBP variability was higher than in recanalized patients. In the whole study population, higher SBP and DBP variability were associated with greater lesion growth on DWI. Interestingly, the correlation between BPV and DWI lesion expansion was higher in patients with persisting occlusion of the medial cerebral artery. In these cases, the correlation of SBP variability (but not of DBP variability) and lesion growth was still present after adjustment for baseline stroke severity, vascular risk factors, localisation of occlusion and the use of antihypertensives. Contrary to this, in patients who recanalized, DWI infarct size was not affected by BPV. Early clinical improvement (defined as a decrease of $\geq 4$ points on the National Institutes of Health stroke scale [NIHSS] score at 24 hours after stroke onset) was correlated with lower BP variability. Moreover, higher SBP variability was associated with higher incidence of poor 3-month outcome defined as a modified Rankin scale (mRS) score $\geq$ 3. The associations of BPV and clinical outcome (early clinical improvement and frequency of 3-month poor outcome) were more evident in the non-recanalized study subjects.

Liu et al studied the impact of systolic BPV on severe haemorrhagic transformation after intravenous thrombolysis [36]. They included 461 patients and measured the following BPV parameters: SD, SV, average squared difference between rise and drop successive measurements (SVrise and SVdrop), and maximum of
SV rise and SV drop (SVrisemax and SVdropmax) after quartering 0-to-24 h BP course. Severe haemorrhagic transformation ( $\mathrm{SHT}$ ) was defined as parenchymal haematoma or symptomatic intracerebral haemorrhage (haemorrhagic transformation and an increase of 4 or more points in NIHSS score). Unfavourable outcome was defined as 2-6 points in mRS at day 90 . They found that SBP-SD and SBP-SV within 24 hours were positively associated with SHT. Within the first 6 hours, only SBP-SV, SBP-SVrise, and SBP-SVrisemax were associated with SHT. Higher SBP-SV, SBP-SVrise, SBP-SVdrop, SBP-SVrisemax, and SBP-SVdropmax among all studies periods $(0-24 \mathrm{~h}, 0-6 \mathrm{~h}, 6-12 \mathrm{~h}, 12-18 \mathrm{~h}, 18-$ $24 \mathrm{~h}$ ) were significantly associated with unfavourable outcomes at day 90 with the exception of SBP-SV drop 0-6 (no relationship).

Endo et al assessed the clinical outcome in stroke patients receiving intravenous thrombolysis $(0.6 \mathrm{mg} /$ $\mathrm{kg}$ dose of rt-PA, recombinant tissue plasminogen activator) and early BPV [23]. In the study, 527 patients were enrolled. BP was measured before administration of rt-PA and every 4 hours after completing the administration (in total, 8 measurements). Then SD, $\mathrm{CV}$, and SV as the square root of the average difference in BP between each of the 8 successive measurements were calculated. Outcomes were as follows: intracerebral haemorrhage within the first 36 hours, modified Rankin Scale (mRS) score of 0 to 1 , and death at 3 months. Higher systolic BPV (SD, CV, SV) was associated with symptomatic intracerebral haemorrhage and death.

In study by Manning et al, a post-hoc analysis of data from two randomized controlled trials (CHHIPS and COSSACS) was undertaken [37]. In COSSACS study, participants with stroke symptom onset $<48$ hours were recruited, while in CHHIPS study, patients with symptom onset $<36$ hours were enrolled. In both studies, baseline (prior to randomization) BP was measured ( 2 sets of 3 BP measurements, 10 minutes apart). The following BPV measures were obtained: SD, CV, ARV, and VIM for SBP and DBP. In both studies, the primary outcome was death or major disability (mRS > 3 at 2 weeks). Regarding COSSACS study, 706 patients were analysed. To assess the relationship between BPV and outcome, adjusted logistic regression was carried out. In the analysis, there was no statistically significant relationship between BPV and death and disability at 2 weeks. In terms of CHHIPS study, no association between short-term BPV and clinical outcome was found.

Tomii et al. evaluated impact of changes of BP on stroke outcome after intravenous (i.v.) thrombolysis [38]. 125 stroke patients who received intravenous rt-PA within 3 hours of symptoms onset were enrolled. 
During the first 24 hours, BP parameters were measured (immediately after hospital admission as well as after starting rt-PA infusion: every 15 minutes during the first 2 hours, every 30 minutes from 2 to 6 hours, and every 1 hour from 6 to 24 hours). Then CV was obtained. The primary outcome was $\mathrm{mRS}<3$ points at 3 months, and the secondary outcome were as follows: early neurological improvement (a reduction of at least 4 points from the baseline NIHSS score or a total NIHSS score of 0 or 1 at 24 hours after i.v. tPA) and intracerebral haemorrhage (found on CT within 36 hours after i.v. rt-PA). In the analysis, CV of SBP and pulse pressure were positively correlated with intracerebral haemorrhage.

In another Japanese study, Tomii et al. investigated the effect 24-hour blood pressure on recovery from AIS. They enrolled 104 ischemic stroke patients admitted to hospital within 24 hours of symptom onset [39]. 24-hour ambulatory BP monitoring was started at 10:00 on the 2 nd and 8th days of hospitalization. Subsequently, CV for SBP, DBP, and MBP was determined. The outcomes were independent activities of daily living ( $\mathrm{mRS} \leq 2$ points) and poor outcome ( $\mathrm{mRS} \geq 5$ points) at 3 months. In multivariate analysis, CV of pulse pressure on the second day was inversely associated with independent activities daily living.

Yong et al. investigated the relationship between BPV and functional outcome, mortality and haemorrhagic complications in 793 patients after acute ischemic stroke included in the ECASS-II study [40]. SV was used to represent BPV. 7-day haemorrhagic transformation and 90-day favourable outcome (mRS score of 0 or 1 point) as well as all-cause mortality were the end points. The results suggest that higher SBP-SV was related to the greater risk of parenchymal haemorrhage $(\mathrm{PH})$ in rtPA-treated group and inversely associated with 90-day favourable outcome in the entire study population. On the other hand, in the placebo-treated participants, there was an inverse association between high SBP-SV and 90-day favourable outcome.

Kellert et al observed 427 patients with acute stroke and investigated the effect of BPV on the development of intracerebral haemorrhage $(\mathrm{ICH})$ after intravenous thrombolysis [41]. BPV was defined as SV and maximum SV for SBP and DBP. Patients were divided into high (> median) and low ( $\leq$ median) systolic and diastolic BPV. ICH was diagnosed on the basis of neuroimaging studies, symptomatic $\mathrm{ICH}(\mathrm{SICH})$ was defined as detection of blood and NIHSS worsening by 4 or more points or leading to death. They found that there were no differences between non- $\mathrm{ICH}, \mathrm{ICH}$ and sICH groups regarding BPV, however patients with high ( $>$ median) SBP-SD had increased risk of occurrence of sICH $(p=0.03)$. Moreover, lower SBP-SD was associa- ted with independent functioning (mRS score $\leq 2$ ) at day $90(p<0.001)$, and mortality at day 90 was more frequent in the high ( $>$ median) diastolic DBP-SD group $(p=0.04)$. The multivariate analysis for the 3-month outcome showed that high systolic BPV was associated with poor outcome.

In a more recent retrospective study of 215 patients de Havenon showed that increased SD, CV, and SV for SBP as well as increased SD and CV for DBP measured within 0-24 hours after admission due to AIS were associated with higher mRS assessed between 30 and 365 days [42]. Only patients with anterior circulation stroke were included in the study. The outcome was worse in the case of proximal artery occlusion and lower mean blood pressure.

In 2004, Castillo et al published their paper on the relationship between SBP, DBP, drop in BP and stroke outcome [43]. Their study included 304 patients with AIS admitted in $<24$ hours after symptom onset. In all patients, BP on admission and on the first day was assessed. Reduction in SBP and DBP > $20 \mathrm{~mm} \mathrm{Hg}$ between the first-day and admission measurements resulted in higher frequency of early neurological deterioration defined as a decrease in the Canadian Stroke Scale (CCS) score $\geq 1$ point within the first 48 hours after admission, increased infarct volume and worse outcome (CCS $\leq 7$ points at 3 months).

In a retrospective study of 2545 ischemic stroke patients by Kang et al, the association of SBP-SD calculated on days 1, 2, and 3 and early neurological deterioration (END, increase of $\geq 2$ points in NIHSS score) at days 1,2 , and 3 was checked [44]. It was shown that higher SBP-SD at day 2 was associated with END at day 2, and SBP-SD at day 3 was associated with END at day 3.

Buratti et al. evaluated 89 ischemic stroke patients with internal carotid artery occlusion [45]. They found that higher values of SD and CV for both SBP and DBP calculated within 48 hours after disease onset were significantly associated with poor outcome assessed at 3 months using the mRS score.

Sare et al. investigated the relationship between blood pressure variability and outcome in ischemic stroke patients admitted to hospital within 8 hours from symptoms onset who received placebo treatment instead of rt-PA [13]. Systolic and diastolic BPV was calculated as CV over 24 and 72 hours. At day 7, neurological impairment (NIHSS > 7); and at day 90, functional outcome (good, $\mathrm{mRS} \leq 2$; and poor, $\mathrm{mRS}>$ 2 ) were obtained. It was shown that (after adjustment for age, sex, time to inclusion, baseline NIHSS, hypertension in history, antihypertensive treatment within the first 7 days) increased systolic BPV was associated with poor 90-day functional outcome but not with early neurological impairment. 


\section{Mid-term blood pressure variability}

Chung et al. evaluated the relationship between BPV and early neurological deterioration (END). In the study, patients with acute ischemic stroke hospitalized within 24 hours after symptoms onset were enrolled [46]. Finally, in the study period (over 3 years), 1161 patients were analysed. Blood pressure was measured for 72 hours of hospitalization. The following blood pressure variability parameters were calculated: standard deviation (SD) and coefficient of variation (CV) for SBP and DBP. END was defined as an increase of at least 2 points in the NIHSS score or increase of at least 1 point in the level of consciousness or motor items of the NIHSS, or the occurrence of new neurological deficits within the first 72 hours after admission to hospital. The study showed that all BPV parameters were higher in patients with END than in patients without END. Furthermore, SBP SD, SBP CV, DBP SD, and DBP $C V$ were linearly associated with END, even after adjustment for mean BP and potential clinical variables (among others, age, sex, history of stroke or transient ischemic attack, diabetes mellitus, atrial fibrillation, Trail of Org 10172 in Acute Stroke Treatment [TOAST] classification, and baseline NIHSS score).

Ko et al assessed relationship between BPV and haemorrhagic transformation (HT) in ischemic stroke patients [47]. The authors enrolled 792 patients hospitalized within 24 hours after stroke onset whose initial gradient echo MRI showed no haemorrhagic transformation. BP was measured during the first 72 hours and the following BPV parameters were obtained: SD and average squared difference between successive measurements (SV) for SBP and DBP. Presence of HT and microbleeds was evaluated on all brain images carried out within first 2 weeks after stroke. The analysis showed that higher DBP SD and DBP-SV were associated with $\mathrm{HT}$. The relationship remained significant for DBP-SD after adjustment for mean SBP, age, time from onset to admission to hospital, initial stroke severity, diabetes mellitus, stroke subtype, thrombolysis, initial glucose, and total cholesterol.

In the TAIST trial, the relationship between BPV defined as SD of SBP and early outcome (at day 10) was assessed [48-49]. In the study, 1479 patients with acute ischemic stroke were randomized. Death or neurologic deterioration (decrease of $\geq 5$ points in the Scandinavian Stroke Scale [SSS] or decrease of $>2$ points in consciousness part of the SSS) and neurologic deterioration alone were associated with increased SBP variability in univariate analysis and after adjustment for baseline prognostic factors, time to treatment and treatment assignment (high-dose tinzaparin, medium-dose tinzaparin, and aspirin). Recurrent stroke (ische- mic, haemorrhagic, or unknown type) was associated with higher SD of SBP in univariate logistic regression, but the relationship lost its statistical significance after adjustment for the aforementioned factors.

In another study by Kang et al, the effect of BPV in the subacute phase of ischemic stroke on the 3-month functional outcome was assessed [50]. 2271 participants hospitalized within 48 hours of symptoms onset were enrolled into this retrospective observational study. The subacute stage was defined as the time period after 72 hours from ischemic stroke symptom onset to discharge or transfer to rehabilitation unit. SD and CV of systolic and diastolic blood pressure were obtained. Clinical outcome was assessed at 3 months using mRS. Poor outcome was defined as a 3-month mRS score of 2 to 6 if the baseline NIHSS score was $\leq 7$ points, mRS score of 3 to 6 if the baseline NIHSS score was 8 to 14 points, and mRS score of 4 to 6 if the baseline NIHSS was $\geq 15$ points. The analysis revealed that patients with poor outcome had a wider range of BP variability than those with good outcome.

A post-hoc analysis of data from ECASS-I study was performed by Yong et al [51]. The correlation between BPV and good functional recovery (mRS score of 0 or 1) at day 90 in ischemic stroke patients was assessed. BPV was defined as SV. Decreased diastolic SV between 0 and 72 hours predicted favourable outcome. Besides, similar relationship was observed for systolic SV, but only in patients treated with rt-PA.

Fukuda et al assessed the relationship between the in-hospital day-by-day blood pressure variability during the acute and subacute stage of ischemic stroke and poor functional outcome at 3 months [52]. They included 2566 patients with acute ischemic stroke and measured BP daily as well as its variability (defined as SD, CV, and VIM). Then the authors evaluated 3-month functional outcome based on mRS. They showed that higher BPV during the subacute stage (but not during the acute) was associated with worse long-term functional outcome after ischemic stroke, independently of age, sex, body mass index, hypertension, diabetes mellitus, dyslipidemia, atrial fibrillation, estimated glomerular filtration rate, ischemic stroke subtype (cardioembolic or non-cardioembolic), infarct area (anterior or posterior circulation), antihypertensive agents administered during days 1-3, the baseline NIHSS score, thrombolytic therapy, and mean BP. Higher SD, CV, and VIM of SBP were also associated with neurological deterioration (defined as an increase in the NIHSS $\geq 2$ during hospitalization) after adjustment for confounding factors.

Tziomalos et al. assessed the relationship between BPV in the acute phase of ischemic stroke and the in-hospital outcome [53]. They evaluated SD and CV of 
SBP and DBP in 608 patients during the first 2 days and the first 3 days after admission to the hospital. The outcome was in-hospital mortality and dependency rate (mRS score between 2 and 5). The authors found no association between BPV and the short-term outcome.

The relationship between BPV measured within 0-72 and 0-120 hours after admission and mRS was analyzed in the aforementioned study by de Havenon et al [42]. It was shown that higher SD, CV, and SV for SBP were predictors of poorer neurological outcome, while BPV for DBP was not significant.

In a prospective study by Wang et al, 873 patients with ischemic stroke were included in the analysis [54]. CV of SBP and CV of DBP calculated for the first 7 days of hospitalization were correlated with good neurological recovery (decrease in NIHSS score by $\geq 4$ points from baseline or 0 point in NIHSS score), neurological deterioration (increase in NIHSS score by $\geq 1$ point), poor functional outcome (2-5 points in mRS score) at 3 months as well as the risk of recurrent stroke, all-cause and cardiovascular mortality, and composite cardiovascular events (cardiovascular mortality, nonfatal stroke, and nonfatal myocardial infarction) within 12 months. They found that increased SBP-CV was significantly associated with less frequent good neurological recovery, neurological deterioration, poor functional outcome, higher risk of recurrent stroke and composite cardiovascular events. The same correlation was found for DBP-CV, except for association with poor functional outcome at 3 months (no correlation).

In another study, de Havenon et al. examined the relationship between SD, CV, and SV of SBP measured within 120 hours after admission in 110 patients with acute anterior circulation ischemic stroke with $\mathrm{mRS}$ score between 30 and 365 days [55]. Higher BPV was found to be associated with worse outcome, especially in patients with larger ischemic core volumes (defined as relative cerebral blood flow $<40 \%$ and absolute arterial tissue delay $>2$ s) and larger hypoperfused volumes (defined as relative mean transit time $>135 \%$ ).

\section{Long-term blood pressure variability}

In a 2014 study, the significance of long-term BPV in patients with ischemic stroke of all subtypes without atrial fibrillation was analyzed [56]. 632 ischemic stroke patients recruited within 14 days of presentation were included in the analysis. The patients were followed up (including BP measurement) every 3-4 months in an outpatient clinic (mean follow-up period was $76 \pm$ 18 months). High BPV was defined as the fourth quartile of the study population. Outcome included all-cause and cardiovascular mortality, non-fatal recurrent stroke and nonfatal acute coronary syndrome. The analysis showed that higher SBP-CV was associated with higher incidence of all-cause and cardiovascular mortality. Furthermore, increased DBP-CV predicted the risk of cardiovascular mortality.

In another study, Lau et al assessed the relationship between visit-to-visit BPV and cardiovascular as well as all-cause mortality in patients with lacunar infarction [57]. 281 patients with lacunar infarction recruited within 14 days of presentation were followed up for $78 \pm 18$ months, on average. During each outpatient clinic visit made every 3-4 months, BP was measured and then systolic and diastolic BPV indices (defined as SD and CV) were calculated. Clinical outcome included all-cause and cardiovascular mortality, recurrent stroke and acute coronary syndrome. The relationship between BPV and clinical characteristics was assessed. Patients with a systolic (but not diastolic) BPV of the third tertile were associated with higher risk of all-cause and cardiovascular mortality. The relationship remained significant after adjustment for age, sex, mean systolic and diastolic BP, cardiovascular risk factors and cardiovascular-related comorbidities.

\section{Meta-analyses}

To our knowledge, only one meta-analysis for the influence of BPV in acute stroke on functional outcome exists was carried out to date [58]. Due to the heterogeneity of observational studies, the analysis included only 7 reports with one study on primary intracerebral haemorrhage [23, 34, 37-39, 50, 59]. It is noteworthy that only variability of BP within the first 7 days following acute stroke was assessed. It was suggested that higher BPV in patients with ischemic stroke is associated with worse functional outcome.

\section{Summary}

BPV was found to be a strong risk factor for stroke and other vascular events in high-risk patients, however little is known about the role of BPV after ischemic stroke [29]. Although some initial reports suggest their potential value in stroke prognosis, to date, few data on this issue are available and the existing results are inconsistent. Generally, the parameter which is most frequently reported as associated with prognosis after ischemic stroke is variability of SBP. In summary, it was shown that its higher values predict death, poor late outcome, haemorrhagic transformation, lesion growth assessed on MRI in case of occluded middle cerebral artery, neurologic deterioration or recurrent stroke $[13,23,35-36,38,41-42,44-48,50-52,54-55$, 
57]. Moreover, higher diastolic BPV was found to be associated with death, haemorrhage, poor short-term outcome and poor late outcome [23, 34-35, 42, 45-47, $50-52,54]$. Only several reports identified higher pulse pressure variability as being associated with haemorrhagic transformation and poor outcome. In one study, it was reported that higher values of mean blood pressure variability were associated with poor late outcome [34, 38-39]. On the other hand, many studies showed no prognostic value of at least some of BPV indices [13, 37, 41-42, 48, 52-54, 56-57].

In a significant majority of studies, variability indices of both systolic and diastolic BP were included $[10,20,30,32,36-37,40-43,45-49,51-52]$, but in four studies [36, 40,44-55] only SBP variability was considered. One study group each evaluated the variability of SBP and PP; SBP, DBP, and PP; and SBP, DBP, and MBP [34, 38-39].

In most studies [10, 29-30,33-35, 39, 43, 46, 49, 52 ], only one BPV index of SBP and/or DBP was evaluated. In several studies, more than one BPV index was assessed (SD and CV in seven studies [36, 41, 45-46, $50,53,56] ; S D, C V$, and SV in three studies [23, 42, 55]; SD and SV in one study [47]; SD, CV, and VIM in one study [52]; and SD, CV, ARV, and VIM in one study [37]). In all of these studies, the importance of assessed indices was similar.

In general, majority of the studies regarding BPV are retrospective $[10,20,31-32,35-37,39,41-43,45-$ $46,50]$, and the number of patients included in these studies is higher than in prospective studies [34-35, 38-39, 45, 52-54, 56-57].

As far as ischemic stroke subtype is concerned, in majority of the studies, this factor was not included in the analysis [10, 20, 31-32,34-37, 39, 43, 46, 48-49]. In several studies, BPV was found to be associated with the outcome after adjustment for, among others, stroke subtype [46-47, 50]. In one study, BPV was not associated with 3-month outcome independently of stroke subtype [38]. In few studies, only one stroke type was assessed: caused by middle cerebral artery occlusion [35] or internal carotid artery occlusion [45], lacunar [57], or anterior circulation ischemic stroke [55]. In one study, all types of stroke in patients without atrial fibrillation were included [56]. In one study, BPV was associated with long-term functional outcome independently of, inter alia, stroke subtype (cardioembolic vs. non-cardioembolic) or infarct area (anterior vs. posterior) [52]. In one study, it was shown that BPV did differ between stroke subtypes (anterior circulation, posterior circulation, or lacunar) and therefore they were not included in further analysis [34].

As demonstrated, studies of the relationship between BPV and outcome in ischemic stroke patients are very heterogeneous; however, the role of BPV seems to be important. Although no clinical trials assessing the effectiveness of BPV reduction in ischemic stroke were carried out to date, it is essential to identify subgroups of patients in whom its influence is highest and who might benefit from such therapies.

\section{References}

1. Lozano R, Naghavi M, Foreman K, Lim S, Shibuya K, Aboyans V, et al. Global and regional mortality from 235 causes of death for 20 age groups in 1990 and 2010: A systematic analysis for the Global Burden of Disease Study 2010. Lancet [Internet]. 2012;380(9859):2095-128. Available from: https://www.thelancet.com/journals/lancet/article/PIIS01406736(12)61728-0/fulltext

2. The 10 leading causes of death [Internet]. [cited 2019 Oct 21]. Available from: https://www.who.int/news-room/factsheets/detail/the-top-10-causes-of-death

3. Global Health Estimates 2014 Summary Tables: DALY BY CAUSE, AGE AND SEX, 2000-2012 [Internet]. [cited 2019 Oct 21]. Available from: https://www.who.int/healthinfo/global burden disease/GHE2016 DALY WHOReg 20002016 .xls?ua=1

4. Chwojnicki K, Ryglewicz D, Wojtyniak B, Zagozdzon P, Czlonkowska A, Jedrzejczyk T, et al. Acute Ischemic stroke hospital admissions, treatment, and outcomes in Poland in 2009-2013. Front Neurol [Internet]. 2018;9:1-9. Available from: https://www.frontiersin.org/articles/10.3389/fneur.2018.00134/full

5. O'Donnell MJ, Denis X, Liu L, Zhang H, Chin SL, Rao-Melacini P, et al. Risk factors for ischaemic and intracerebral haemorrhagic stroke in 22 countries (the INTERSTROKE study): A case-control study. Lancet [Internet]. 2010;376(9735):112-23. Available from: https://www.thelancet.com/journals/lancet/article/PIIS0140-6736(10)60834-3/fulltext

6. Kotlęga D, Gołąb-Janowska M, Meller A, Pawlukowska W, Nowacki P. Detection of stroke risk factors over the decade in the polish population of ischemic stroke patients. Adv Psychiatry Neurol [Internet]. 2019;28(2):83-7. Available from: https://www.termedia.pl/Wykrywalnosc-czynnikow-ryzyka-udaru-na-przestrzeni-dekady-w-polskiej-populacji-pacjentow-z-udarem-niedokrwiennym-mozgu,116,37026,1,0.html 
7. Swarowska M, Burkot J, Janowska A, Klimkowicz-Mrowiec A, Pera J, Slowik A, et al. Improvement of survival in Polish stroke patients is related to reduced stroke severity and better control of risk factors: The Krakow Stroke Database. Arch Med Sci [Internet]. 2016;12(3):552-5. Available from: https://www.ncbi.nlm.nih.gov/pmc/articles/PMC4889689/pdf/ AMS-12-27569.pdf

8. Zhang H, Thijs L, Staessen JA. Blood pressure lowering for primary and secondary prevention of stroke. Hypertension [Internet]. 2006;48(2):187-95. Available from: https://www.ahajournals.org/doi/10.1161/01.HYP.0000231939.40959.60

9. Aiyagari V, Gorelick PB. Management of blood pressure for acute and recurrent stroke. Stroke [Internet]. 2009;40(6):22516. Available from: https://www.ahajournals.org/doi/10.1161/STROKEAHA.108.531574

10. Ringleb PA, Bousser M, Ford G, Bath P, Brainin M, Caso V, et al. Guidelines for management of ischaemic stroke and transient ischaemic attack 2008. Cerebrovasc Dis [Internet]. 2008;25(5):457-507. Available from: https://www.karger.com/ Article/FullText/131083

11. Stead LG, Gilmore RM, Decker WW, Weaver AL, Brown RD. Initial emergency department blood pressure as predictor of survival after acute ischemic stroke. Neurology [Internet]. 2005;65(8):1179-83. Available from: https://n.neurology.org/content/65/8/1179

12. Aslanyan S, Fazekas F, Weir CJ, Horner S, Lees KR. Effect of blood pressure during the acute period of ischemic stroke on stroke outcome: A tertiary analysis of the GAIN International Trial. Stroke [Internet]. 2003;34(10):2420-5. Available from: https://www.ahajournals.org/doi/10.1161/01.STR.0000091233.04524.0C

13. Sare GM, Ali M, Shuaib A, Bath PMW. Relationship between hyperacute blood pressure and outcome after ischemic stroke: data from the VISTA collaboration. Stroke [Internet]. 2009 Jun;40(6):2098-103. Available from: https://www. ahajournals.org/doi/10.1161/STROKEAHA.108.539155

14. Tikhonoff V, Zhang H, Richart T, Staessen J a. Blood pressure as a prognostic factor after acute stroke. Lancet Neurol [Internet]. 2009;8(10):938-48. Available from: https://www.thelancet.com/journals/laneur/article/PIIS1474-4422(09)70184-X/fulltext

15. Mancia G, Fagard R, Narkiewicz K, Redon J, Zanchetti A, Böhm M, et al. 2013 ESH/ESC guidelines for the management of arterial hypertension: The Task Force for the management of arterial hypertension of the European Society of Hypertension (ESH) and of the European Society of Cardiology (ESC). Eur Heart J [Internet]. 2013;34(28):2159-219. Available from: https://academic.oup.com/eurheartj/article/34/28/2159/451304

16. Rodríguez-García JL, Botia E, De La Sierra A, Villanueva MA, González-Spínola J. Significance of elevated blood pressure and its management on the short-term outcome of patients with acute ischemic stroke. Am J Hypertens [Internet]. 2005;18(3):379-84. Available from: https://academic.oup.com/ajh/article/18/3/379/160939

17. Ntaios G, Lambrou D, Michel P. Blood pressure changes in acute ischemic stroke and outcome with respect to stroke etiology. Neurology [Internet]. 2012;79:1440-8. Available from: https://n.neurology.org/content/79/14/1440

18. Okumura K, Ohya Y, Maehara A, Wakugami K, Iseki K, Takishita S. Effects of blood pressure levels on case fatality after acute stroke. J Hypertens [Internet]. 2005 Jun;23(6):1217-23. Available from: https://insights.ovid.com/crossref?an=00004872-200506000-00017

19. Harper G, Castleden CM, Potter JF, G. H, C.M. C, J.F. P. Factors affecting changes in blood pressure after acute stroke. Stroke [Internet]. 1994;25(9):1726-9. Available from: https://www.ahajournals.org/doi/10.1161/01.STR.25.9.1726

20. Qureshi Al. Acute hypertensive response in patients with stroke pathophysiology and management. Circulation [Internet]. 2008;118(2):176-87. Available from: https://www.ahajournals.org/doi/10.1161/CIRCULATIONAHA.107.723874

21. Christensen H, Meden P, Overgaard K, Boysen G. The course of blood pressure in acute stroke is related to the severity of the neurological deficits. Acta Neurol Scand [Internet]. 2002;106(3):142-7. Available from: https://onlinelibrary.wiley. com/doi/full/10.1034/i.1600-0404.2002.01356.x

22. Rothwell PM. Limitations of the usual blood-pressure hypothesis and importance of variability, instability, and episodic hypertension. Lancet [Internet]. 2010;375(9718):938-48. Available from: https://www.thelancet.com/journals/lancet/ article/PIIS0140-6736(10)60309-1/fulltext

23. Endo K, Kario K, Koga M, Nakagawara J, Shiokawa Y, Yamagami H, et al. Impact of early blood pressure variability on stroke outcomes after thrombolysis: The SAMURAI rt-PA registry. Stroke [Internet]. 2013;44(3):816-8. Available from: https://www.ahajournals.org/doi/10.1161/STROKEAHA.112.681007

24. Webb AJ, Fischer U, Mehta Z, Rothwell PM. Effects of antihypertensive-drug class on interindividual variation in blood pressure and risk of stroke: a systematic review and meta-analysis. Lancet [Internet]. 2010;375(9718):906-15. Available from: https://www.thelancet.com/journals/lancet/article/PIIS0140-6736(10)60235-8/fulltext

25. Kikuya M, Ohkubo T, Metoki H, Asayama K, Hara A, Obara T, et al. Day-by-day variability of blood pressure and heart rate at home as a novel predictor of prognosis: The Ohasama study. Hypertension [Internet]. 2008;52(6):1045-50. Available from: https://www.ahajournals.org/doi/10.1161/HYPERTENSIONAHA.107.104620

26. Parati G, Ochoa JE, Lombardi C, Bilo G. Assessment and management of blood-pressure variability. Nat Rev Cardiol [Internet]. 2013;10(3):143-55. Available from: http://www.ncbi.nlm.nih.gov/pubmed/23399972 
27. Stergiou GS, Parati G, Vlachopoulos C, Achimastos A, Andreadis E, Asmar R, et al. Methodology and technology for peripheral and central blood pressure and blood pressure variability measurement. J Hypertens [Internet]. 2016;34(9):166577. Available from: https://insights.ovid.com/crossref?an=00004872-201609000-00002

28. Stevens SL, Wood S, Koshiaris C, Law K, Glasziou P, Stevens RJ, et al. Blood pressure variability and cardiovascular disease: systematic review and meta-analysis. BMJ [Internet]. 2016;354:i4098. Available from: https://www.bmj.com/content/354/bmj.i4098

29. Rothwell PM, Howard SC, Dolan E, O'Brien E, Dobson JE, Dahlöf B, et al. Prognostic significance of visit-to-visit variability, maximum systolic blood pressure, and episodic hypertension. Lancet [Internet]. 2010;375(9718):895-905. Available from: https://www.thelancet.com/journals/lancet/article/PIIS0140-6736(10)60308-X/fulltext

30. Webb AJS, Fischer U, Rothwell PM. Effects of beta-blocker selectivity on blood pressure variability and stroke: A systematic review. Neurology [Internet]. 2011;77(8):731-7. Available from: https://n.neurology.org/content/77/8/731

31. Parati G , Faini A VM. Blood pressure variabiliy: Its measurement and significance in hypertension. Curr Hypertens Rep [Internet]. 2006;8:199-204. Available from: https://www.nature.com/articles/nrcardio.2013.1

32. Parati G, Ochoa JE, Salvi P, Lombardi C, Bilo G. Prognostic value of blood pressure variability and average blood pressure levels in patients with hypertension and diabetes. Diabetes Care [Internet]. 2013;36(SUPPL.2):S3212-324. Available from: https://care.diabetesjournals.org/content/diacare/36/Supplement 2/S312.full.pdf

33. Chadachan VM, Ye MT, Tay JC, Subramaniam K, Setia S. Understanding short-term blood-pressure-variability phenotypes: From concept to clinical practice. Int J Gen Med [Internet]. 2018;11:241-54. Available from: https://www.dovepress. com/understanding-short-term-blood-pressure-variability-phenotypes-from-co-peer-reviewed-fulltext-article-IJGM

34. Dawson SL, Manktelow BN, Robinson TG, Panerai RB, Potter JF. Which parameters of beat-to-beat blood pressure and variability best predict early outcome after acute ischemic stroke? Stroke. 2000;31(2):463-8.

35. Delgado-Mederos R, Ribo M, Rovira A, Rubiera M, Manuera J, Santamarina E, et al. Prognostic significance of blood pressure variability after thrombolysis in acute stroke. Neurology [Internet]. 2008;71(8):552-8. Available from: https://n. neurology.org/content/71/8/552

36. Liu K, Yan S, Zhang S, Guo Y, Lou M. Systolic blood pressure variability is associated with severe hemorrhagic transformation in the early stage after thrombolysis. Transl Stroke Res [Internet]. 2016;7(3):186-91. Available from: https://link. springer.com/article/10.1007\%2Fs12975-016-0458-6

37. Manning LS, Mistri AK, Potter J, Rothwell PM, Robinson TG. Short-Term Blood Pressure Variability in Acute Stroke: Post hoc analysis of the controlling hypertension and hypotension immediately post stroke and continue or stop post-stroke antihypertensives collaborative study trials. Stroke [Internet]. 2015;46(6):1518-24. Available from: https://www.ahajournals.org/doi/10.1161/STROKEAHA.115.009078

38. Tomii Y, Toyoda K, Nakashima T, Nezu T, Koga M, Yokota C, et al. Effects of hyperacute blood pressure and heart rate on stroke outcomes after intravenous tissue plasminogen activator. J Hypertens [Internet]. 2011;29(10):1980-7. Available from: https://insights.ovid.com/crossref?an=00004872-201110000-00020

39. Tomii Y, Toyoda K, Suzuki R, Naganuma M, Fujinami J, Yokota C, et al. Effects of 24-hour blood pressure and heart rate recorded with ambulatory blood pressure monitoring on recovery from acute ischemic stroke. Stroke [Internet]. 2011;42(12):3511-7. Available from: https://www.ahajournals.org/doi/10.1161/STROKEAHA.111.628586

40. Yong M, Kaste M. Association of characteristics of blood pressure profiles and stroke outcomes in the ECASS-II trial. Stroke [Internet]. 2008 Mar;39(2):366-72. Available from: https://www.ahajournals.org/doi/10.1161/STROKEAHA.107.492330

41. Kellert L, Sykora M, Gumbinger C, Herrmann O, Ringleb P a. Blood pressure variability after intravenous thrombolysis in acute stroke does not predict intracerebral hemorrhage but poor outcome. Cerebrovasc Dis [Internet]. 2012;33(2):13540. Available from: https://www.karger.com/Article/FullText/334186

42. de Havenon A, Bennett A, Stoddard GJ, Smith G, Wang H, Wold J, et al. Increased blood pressure variability is associated with worse neurologic outcome in acute anterior circulation ischemic stroke. Stroke Res Treat [Internet]. 2016;2016:1-8. Available from: https://www.hindawi.com/journals/srt/2016/7670161/

43. Castillo J, Leira R, García MM, Serena J, Blanco M, Dávalos A. Blood pressure decrease during the acute phase of ischemic stroke is associated with brain injury and poor stroke outcome. Stroke [Internet]. 2004;35(2):520-6. Available from: https://www.ahajournals.org/doi/10.1161/01.STR.0000109769.22917.BO

44. Kang J, Hong J-H, Jang MU, Choi NC, Lee JS, Kim BJ, et al. Change in blood pressure variability in patients with acute ischemic stroke and its effect on early neurologic outcome. PLoS One [Internet]. 2017;12(12):e0189216. Available from: http://dx.plos.org/10.1371/journal.pone.0189216

45. Buratti L, Cagnetti C, Balucani C, Viticchi G, Falsetti L, Luzzi S, et al. Blood pressure variability and stroke outcome in patients with internal carotid artery occlusion. J Neurol Sci [Internet]. 2014;339(1-2):164-8. Available from: https://www. ins-journal.com/article/S0022-510X(14)00093-8/fulltext 
46. Chung J-W, Kim N, Kang J, Park SH, Kim W-J, Ko Y, et al. Blood pressure variability and the development of early neurological deterioration following acute ischemic stroke. J Hypertens [Internet]. 2015;33(10):2099-106. Available from: https:// insights.ovid.com/article/00004872-201510000-00020

47. Ko Y, Park JH, Yang MH, Ko SB, Han MK, Oh CW, et al. The significance of blood pressure variability for the development of hemorrhagic transformation in acute ischemic stroke. Stroke [Internet]. 2010;41(11):2512-8. Available from: https:// www.ahajournals.org/doi/10.1161/STROKEAHA.110.595561

48. Geeganage C, Tracy M, England T, Sare G, Moulin T, Woimant F, et al. Relationship between baseline blood pressure parameters (including mean pressure, pulse pressure, and variability) and early outcome after stroke: Data from the tinzaparin in acute ischaemic stroke trial (TAIST). Stroke [Internet]. 2011;42(2):491-3. Available from: https://www.ahajournals.org/doi/10.1161/STROKEAHA.110.596163

49. Bath PMW, Lindenstrom E, Boysen G, De Deyn P, Friis P, Leys D, et al. The Tinzaparin in Acute Ischaemic Stroke Trial stroke (TAIST): a randomised aspirin-controlled trial. Hypertension [Internet]. 2001;358:702-10. Available from: https://www. thelancet.com/iournals/lancet/article/PIIS0140-6736(01)05837-8/fulltext

50. Kang J, Ko Y, Park JH, Kim WJ, Jang MS, Yang MH, et al. Effect of blood pressure on 3-month functional outcome in the subacute stage of ischemic stroke. Neurology [Internet]. 2012;79(20):2018-24. Available from: https://n.neurology.org/ content/79/20/2018

51. Yong M, Diener H-C, Kaste M, Mau J. Characteristics of blood pressure profiles as predictors of long-term outcome after acute ischemic stroke. Stroke [Internet]. 2005;36(12):2619-25. Available from: https://www.ahajournals.org/ doi/10.1161/01.STR.0000189998.74892.24

52. Fukuda K, Kai H, Kamouchi M, Hata J, Ago T, Nakane H, et al. Day-by-Day Blood Pressure Variability and Functional Outcome After Acute Ischemic Stroke: Fukuoka Stroke Registry. Stroke [Internet]. 2015;1832-9. Available from: https://www. ahajournals.org/doi/10.1161/STROKEAHA.115.009076

53. Tziomalos K, Giampatzis V, Bouziana SD, Spanou M, Kostaki S, Papadopoulou M, et al. No association observed between blood pressure variability during the acute phase of ischemic stroke and in-hospital outcomes. Am J Hypertens [Internet]. 2015;29(7):841-846. Available from: http://ajh.oxfordjournals.org/lookup/doi/10.1093/ajh/hpv191

54. Wang Y, Meng P, Liu N, Ji N, Zhang G, Xu B, et al. Mid-term blood pressure variability Is associated with clinical outcome after ischemic stroke. Am J Hypertens [Internet]. 2017;301(10):968-77. Available from: https://academic.oup.com/ajh/ article/30/10/968/3867193

55. de Havenon A, Bennett A, Stoddard GJ, Smith G, Chung L, O'Donnell S, et al. Determinants of the impact of blood pressure variability on neurological outcome after acute ischaemic stroke. Stroke Vasc Neurol [Internet]. 2017;2(1):1-6. Available from: $\mathrm{http}: / /$ svn.bmj.com/cgi/doi/10.1136/svn-2016-000057

56. Lau K-K, Wong Y-K, Teo K-C, Chang RSK, Chan K-H, Hon SFK, et al. Long-term prognostic implications of visit-to-visit blood pressure variability in patients with ischemic stroke. Am J Hypertens [Internet]. 2014;27(12):1486-94. Available from: https://academic.oup.com/ajh/article/27/12/1486/2743148

57. Lau KK, Wong YK, Chang RSK, Teo KC, Hon SFK, Chan KH, et al. Visit-to-visit systolic blood pressure variability predicts all-cause and cardiovascular mortality after lacunar infarct. Eur J Neurol [Internet]. 2014;21(2):319-25. Available from: https://onlinelibrary.wiley.com/doi/full/10.1111/ene.12310

58. Manning LS, Rothwell PM, Potter JF, Robinson TG. Prognostic significance of short-term blood pressure variability in acute stroke. Stroke [Internet]. 2015;46(9):2482-90. Available from: http://stroke.ahajournals.org/lookup/doi/10.1161/ STROKEAHA.115.010075

59. Manning L, Hirakawa Y, Arima H, Wang X, Chalmers J, Wang J, et al. Blood pressure variability and outcome after acute intracerebral haemorrhage: a post-hoc analysis of INTERACT2, a randomised controlled trial. Lancet Neurol [Internet]. 2014 Apr [cited 2015 Dec 19];13(4):364-73. Available from: https://www.thelancet.com/journals/laneur/article/ PIIS1474-4422(14)70018-3/fulltext 